\title{
THE NOAO CCD CONTROLLER - ARCON
}

\author{
Alistair R. Walker \\ Cerro Tololo Inter-American Observatory,
}

The Array Controller (Arcon) developed at CTIO is a flexible and extendable system for acquiring astronomical data from a wide variety of optical and IR detectors. It has been designed from the outset to support expansion to more demanding applications such as large CCD mosaics. In its minimum configuration it can read a quad-readout CCD through all four amplifiers simultaneously.

To provide real time performance and portability, most of the software executes within the Arcon, rather than the host, in a network of parallel processors (Inmos Transputers) which is easily extended as more demanding applications arise. Transputers (rather than DSPs) were chosen since they provide excellent hardware support for real time programs (e.g., rapid scheduling instructions, timers, eight DMA driven I/O channels), a simple, efficient and uniform inter-process and inter-processor communication mechanism, unified software development and debugging tools for the entire processor network, and a wide range of standardized, compact and relatively low power hardware subsystems from a variety of third party vendors.

The entire controller is compact enough to be housed in a single small enclosure permanently attached to the CCD dewar, connected only to AC power and a pair of optical fibers carrying commands, status and data to a small network of transputers ("Tram box") located near a SUN workstation. These commercial transputer modules are interfaced to the SUN by a single Sbus card. Most of the Arcon software runs in an Inmos transputer in the Tram box. This processor is dubbed the "Nexus" since it is the communications hub of the system. It accepts high level requests from the host and generates the appropriate subsystem commands and status requests to set-up the detector, run waveforms, and read back image data. It assembles header information in FITS format and stores the incoming image in a $2.5 \mathrm{MB}$ software FIFO, plenty large enough to absorb the worst case Unix latencies. Another transputer serves as an intelligent display controller which presents a 1280 × 1024 image at full resolution as it is read out, performing extensive real time processing to allow all quadrants to be viewed with the same intensity scaling, while removing the overscan from the center of the image, orienting it to match finding charts and marking saturated pixels in red.

The IRAF-based user interface in the Unix workstation seamlessly integrates data acquisition and reduction. Observational parameters are set using the familiar IRAF parameter editor (epar). Data acquisition, telescope and instrument commands may be mixed with normal IRAF commands in CL scripts. Scripts have been provided which automate observing operations such as focusing and exposure sequences through various filters, as well as performing CCD characterization and controller diagnostics. Status information is divided into several windows so the observer can tailor the information displayed as desired. Both status 
windows and control windows may be opened or duplicated on consoles or terminals anywhere on the network. This versatility allows for efficient use of the system by single or multiple observers, and enables remote diagnostics by staff not present at the telescope.

A Waveform Definition Language (WDL) compiler and plotting software have been developed, which make it practical to customize waveforms for each detectors. WDL allows run time control of characteristics which were formerly considered to be "hard wired", such as output amplifier selection. It also allows the automation and acceleration of engineering tests which have formerly been prohibitively time consuming.

Other unusual features of the hardware are very low power consumption, DC coupled video to improve overscan behavior, the widespread use of surface mount technology, built-in test signal and data generators and extensive self-diagnostics, and a modularization which allows board replacement without manual adjustment.

Arcon controllers are now responsible for taking the majority of CCD data at CTIO, with implementations onto Thomson $1 \mathrm{k}$, Tektronix $1 \mathrm{k}$ and $2 \mathrm{k}$ CCDs and a Loral $3 \mathrm{k}$ CCD. Most of these CCDs have four output amplifiers; as an example, with a Tektronix $2 \mathrm{k}$ CCD it takes 30 seconds from initiation of readout to having the image stored on disk, in IRAF format, with $4.5 \mathrm{e}^{-}$read noise. Experience has shown that the Arcon controllers are reliable, robust and immune to interference, with scientific performance greatly superior to that of CTIO's previous generation of controllers. The system has proven popular and easy to learn. We are presently converting our remaining CCDs to Arcon controllers. Meanwhile the existing controllers at KPNO are being upgraded with the Arcon software and digital hardware, and the full Arcon has been adopted for new applications such as the WIYN telescope, and the Gemini 8-m telescopes. An expanded version of the controller will be used to operate the $8 \mathrm{k} \mathrm{x} 8 \mathrm{k}$ mosaic array (eight CCDs, 16 amplifiers) presently under development at NOAO.

The building of Arcon controllers has been a team effort: Tom Ingerson (project manager), Alistair Walker (project scientist), Roger Smith, Rolando Rogers, Eduardo Mondaca, Andy Rudeen (hardware), Dan Smith, Gary Webb, Jim Hughes, Pedro Gigoux and Steve Heathcote (software) have all made major contributions. But principally, the Arcon controller owes much of its success to the vision, the technical proficiency, and the driving enthusiasm of Roger Smith. 